\title{
Determination of thoracic epidural catheter placement: electrical epidural stimulation (Tsui test) is simple, effective, and under- utilized
}

\author{
M. Kwesi Kwofie, MD • Gordon Launcelott, MD • Ban C. H. Tsui, MSc, MD
}

Received: 12 December 2018/Revised: 18 December 2018/Accepted: 20 December 2018/Published online: 23 January 2019

(C) Canadian Anesthesiologists' Society 2019

The incidence of failure to achieve adequate postoperative analgesia via a thoracic epidural catheter has been reported to exceed $30 \% .^{1,2}$ This speaks to the difficulty of assessing correct catheter placement in the epidural space that directly relates to its function. Bearing this in mind, we were pleased to read the article in this issue of the Journal, by Balki et al., ${ }^{3}$ which compared the agreement between the responses with a standard local anesthetic test dose and with the epidural electrical stimulation test (EEST) in patients undergoing abdominal surgery. The study demonstrated that the EEST is a sensitive and effective test for confirming the correct position of a thoracic epidural catheter, which relates to its ability to provide adequate postoperative epidural analgesia.

With the EEST (also known as a "Tsui test"), a continuous column of saline through a common coilreinforced catheter connects the catheter tip to a stimulator that delivers controlled, reproducible electrical stimulation of axons contained in the epidural space. The ability to evoke motor responses (muscle contractions) reflects the correct positioning of the catheter in the epidural space, and the muscles that contract relate to the spinal level of catheter placement. In point of fact, the demonstrable reliability of the EEST is not new. Since its initial

M. K. Kwofie, MD · G. Launcelott, MD

Department of Anesthesia, Perioperative Medicine and Pain

Management, Dalhousie University, Halifax, NS, Canada

B. C. H. Tsui, MSc, MD ( $ه)$

Department of Anesthesiology, Perioperative and Pain Medicine, Stanford University School of Medicine, 300 Pasteur Drive,

Stanford, CA, USA

e-mail: bantsui@stanford.edu description in 1998 by Tsui et al., ${ }^{4}$ multiple studies in adult surgical patients have verified the high sensitivity of the test to confirm correct lumbar and thoracic epidural catheter position, ranging between 80 and $100 \% .^{4-7}$ Use of the test has been extended to include pediatric patients to help identify correct position of caudally inserted epidural catheters threaded rostrally into the thoracic epidural space. ${ }^{8}$

Given the demonstrated value of the EEST, it is somewhat surprising that this technique has not become more widely adopted. All the more so considering that electrical stimulation has long since become an accepted strategy to help identify the correct position of catheter placement and local anesthetic deposition for peripheral nerve blocks. At Dalhousie University, one of the authors (G.L.) was an early advocate for the utility of the EEST to assess correct thoracic epidural catheter placement and with nearly two decades of experience can say with confidence that this it is easy, practical, and safe. In fact, at hospitals affiliated with Dalhousie University, anesthesia technicians are specifically trained to assist with the EEST including the stocking and maintenance of the appropriate equipment on all of the portable epidural equipment carts.

Much effort continues to be spent assessing other techniques to confirm correct epidural catheter tip positioning, including fluoroscopy and epidural pressure waveform analysis. However, none enjoy broad clinical use yet. Fluoroscopy with epidurogram is a highly effective technique to reliably guide and confirm correct needle or catheter positioning in the epidural space. This technique can also confirm the injectate level and spread and is widely used in the management of chronic pain. Nevertheless, radiation exposure, availability, and the added cost and time have limited the widespread use of fluoroscopy in the perioperative setting. 
Ultrasound-guided needle and catheter insertion has become a standard of practice for performing peripheral regional anesthesia. In contrast, the popularity of ultrasound for neuraxial procedures has been more muted. In pediatric subjects, neuraxial structures are easily identified allowing real-time visualization of interlaminar needle passage and injectate spread. ${ }^{9}$ In adults, however, the increased calcification of bone limits realtime needle guidance, but in the lumbar space, ultrasound can be useful as a pre-procedural scan to decrease the number of needle passes in patients with difficult anatomy. ${ }^{10}$ In the adult mid-thoracic spine, the relatively narrow inter-laminar spaces and steep thoracic spinous processes frequently limit insonation of structures deep to the lamina. Though it has been suggested that preprocedural sonography of the thoracic spine can provide clinical benefit by defining the spinal anatomy, ${ }^{11}$ this advantage was refuted in a recent randomized trial. ${ }^{12}$ Doppler ultrasound has recently been shown to help visualize epidural catheters placed in the lumbar epidural space; nevertheless, efficacy of this technique has not been demonstrated in the thoracic spine. ${ }^{13}$

Epidural waveform analysis has been used to confirm the presence of the epidural needle or catheter in the epidural space. ${ }^{14}$ On the plus side, this technique is relatively inexpensive and simple to perform with equipment that is readily available. Nonetheless, the factors governing the relationship between epidural waveform and catheter/nerve position are relatively obscure and in some cases the waveform can be difficult to assess. In addition, epidural waveform analysis does not differentiate catheter tip position with regard to the epidural, intrathecal, or vascular spaces. Finally, the technique does not indicate spinal segment level or laterality of catheter tip position.

The local anesthetic test dose is considered by many to be the gold standard by which correct epidural catheter positioning should be assessed. In theory, the test dose can also help to diagnose a catheter that is inserted in the intrathecal or intravascular space. Nevertheless, the assessment of sensory block demands a cooperative patient, requires adequate time to allow a drug effect, and relies on the patient's subjective response, which may not be accurate.

Compared with these other modalities, the EEST is relatively simple, efficient, and portable and is not associated with radiation exposure to patients or practitioners. The EEST has broad diagnostic capability and has many benefits: it is sensitive ${ }^{4-7}$; can be rapidly administered; has a reproducible and objective outcome (visible motor-evoked response); can determine the level of the epidural catheter tip; can differentiate catheters that are positioned subcutaneously, intrathecally, or intravascularly ${ }^{15}$; and may be able to identify a subdural catheter position $^{16,17}$ (but this is controversial). ${ }^{18}$

In our opinion, the advantages of the EEST make it a superior test for the confirmation of correct epidural catheter positioning. When patient care is handed off from the OR anesthesiologist to another postoperative acute pain service physician/team, it is particularly helpful to have a documented objective assessment of the epidural catheter position at installation. After all, if the EEST is so useful, why, after 20 years since its description, has it not become more widely employed?

We speculate that many anesthesiologists are reluctant to accept the high failure rate of epidural analgesia reported in the literature and continue to practice under the misguided belief that a successful "loss of resistance technique" and uneventful epidural catheter threading are sufficient evidence for correct catheter positioning. Another issue is the time pressure "to get on with the case", which impedes the adoption of tests to assess the epidural catheter's position. A challenge particular to the EEST is the availability of appropriate equipment. In particular, most nerve stimulators are not equipped with the necessary features. These include a current range of $0.1-15$ $\mathrm{mA}$, pulse width of 0.1 to $0.2 \mathrm{msec}$, pulse frequency of $1-2$ $\mathrm{Hz}$, digital current read out, and appropriate connectors (anode to an electrocardiogram [ECG] electrode, cathode to a Johan's ECG adaptor). We would draw readers' attention to the fact that the originally described range of current required to evoke a motor response, $1-10 \mathrm{~mA},{ }^{2}$ has been expanded to $1-15 \mathrm{~mA} .{ }^{19}$ In contrast, with little effort, routinely used epidural catheters can be employed for the EEST. It is essential to create a continuous column of conducting fluid within the stainless-steel wire-reinforced epidural catheter by priming it with saline after insertion and to ensure that there is no air within the system. ${ }^{3} \mathrm{We}$ have found that application of gentle continuous pressure on the saline column during stimulation facilitates a reliable and repeatable evoked motor response. It is notable that most studies evaluating the test do not use a stimulating catheter. $^{3-5}$ If using a stimulating epidural catheter, saline priming is not required. ${ }^{20}$

The EEST has been criticized for its inability to identify a catheter tip located in the paravertebral space. ${ }^{4}$ Aside from continuous fluoroscopy with epidurography, no other method has yet been shown to resolve this issue, though it would seem biologically plausible that epidural waveform analysis may be of benefit. Another issue to consider is that some degree of epidural analgesia may be still achieved with catheters placed outside of the epidural space when sufficient volumes of local anesthetic are administered, as observed with interfascial plane blocks (e.g., erector spinae plane, ${ }^{21}$ retrolaminar, ${ }^{22}$ and quadratus lumborum blocks). ${ }^{23}$ In such a case, the inability to evoke a motor response 
using the EEST would not correspond with an epidural analgesic effect and so would be interpreted as a "false negative". Notwithstanding, we would emphasize that the EEST would have correctly alerted that the catheter was not inserted into the epidural space.

After 20 years since its initial description, the EEST, or Tsui test, remains an objective, portable, reliable, and safe technique that may help to decrease the high rate of epidural catheter failure. Though the adoption of the EEST in adults appears to be quite slow, the clinical need to decrease thoracic epidural block failure is well documented. We hope that the study by Balki et al. ${ }^{3}$ will remind clinicians that the EEST is a superior option when objective confirmation of epidural catheter positioning is sought. We are confident that routine adoption of the EEST will help to maximize the benefits derived from epidural analgesia.

\section{Détermination du positionnement d'un cathéter péridural thoracique : la stimulation électrique péridurale (test de Tsui) est simple, efficace et sous-utilisée}

L'incidence de l'échec d'une analgésie postopératoire adéquate au moyen d'un cathéter péridural thoracique dépasse $30 \%$ selon certaines publications. ${ }^{1,2}$ Cela témoigne de la difficulté à évaluer correctement la position du cathéter dans l'espace péridural directement en rapport avec son utilisation. Ayant cela en tête, nous avons été heureux de lire l'article de Balki et coll. $^{3}$ dans ce numéro du Journal. Les auteurs y ont comparé la concordance des réponses avec une dose test d'un anesthésique local usuelle avec celles d'un test de stimulation électrique péridurale (EEST) chez des patients subissant une chirurgie abdominale. L'étude a montré que l'EEST est une méthode efficace et sensible de confirmation du positionnement correct d'un cathéter péridural thoracique, pour ce qui concerne son aptitude à procurer une analgésie péridurale postopératoire adéquate.

Avec l'EEST (également connu sous le nom de « test de Tsui »), une colonne continue de salin passant à travers un cathéter renforcé par un filage relie l'extrémité du cathéter à un stimulateur qui délivre une stimulation électrique contrôlée et reproductible des axones présents dans l'espace péridural. L'aptitude à déclencher des réponses motrices (contractions musculaires) témoigne du bon positionnement du cathéter dans l'espace péridural et les muscles qui se contractent correspondent au niveau vertébral de l'emplacement du cathéter. De fait, la démonstration de la fiabilité de l'EEST n'est pas nouvelle. Depuis sa première description par Tsui et coll. $^{4}$ en 1998, de nombreuses études menées chez les patients chirurgicaux adultes ont vérifié la grande sensibilité du test pour confirmer le bon positionnement lombaire et thoracique du cathéter allant de $80 \%$ à $100 \%$ des cas. ${ }^{4-7}$ L'utilisation de ce test a été élargie aux patients pédiatriques pour contribuer à identifier le on positionnement de cathéters épiduraux insérés en position caudale et poussés en direction céphalade dans l'espace péridural thoracique. $^{8}$

Compte tenu de la valeur démontrée de l'EEST, il est assez surprenant que cette technique n'ait pas été plus largement adoptée. D'autant plus que si on considère que la stimulation électrique est devenue - depuis longtemps une stratégie d'identification acceptée du bon positionnement du cathéter et de dépôt d'un anesthésique local pour les blocs nerveux périphériques. À l'université Dalhousie, l'un des auteurs (G.L.) a été très tôt un ardent défenseur de la place de l'EEST pour évaluer le bon positionnement d'un cathéter péridural thoracique; avec maintenant presque 20 ans d'expérience, on peut dire sans crainte que la méthode est facile, pratique et sécuritaire. Dans les hôpitaux affiliés à l'université Dalhousie, les techniciens en anesthésie sont formés spécialement pour aider l'EEST, y compris en veillant au stockage et à l'entretien de l'équipement approprié sur tous leurs chariots de matériel pour péridurales.

Beaucoup d'efforts continuent à être déployés pour évaluer d'autres techniques visant à confirmer le bon positionnement de l'extrémité du cathéter péridural, notamment la fluoroscopie et l'analyse du tracé de la pression péridurale. Cependant, aucune de ces méthodes n'a encore été largement utilisée en clinique. La fluoroscopie avec un péridurogramme est une technique très efficace pour guider de manière fiable et confirmer le bon positionnement de l'aiguille ou du cathéter dans l'espace péridural. Cette technique peut aussi confirmer le niveau pour l'injection et son étendue; elle est largement utilisée dans la gestion de la douleur chronique. Néanmoins, l'exposition aux radiations, sa disponibilité, et le coût supplémentaire, ainsi que le temps requis ont limité la généralisation de la fluoroscopie dans un contexte périopératoire.

L'insertion sous échographie de l'aiguille et du cathéter est devenue une norme dans la réalisation de l'anesthésie régionale périphérique. En revanche, l'enthousiasme de l'échographie pour les procédures neuraxiales est plus mitigé. Chez les patients pédiatriques, les structures neuraxiales sont facilement identifiées, permettant une 
visualisation en temps réel du passage de l'aiguille entre les lames et de la diffusion du produit injecté. ${ }^{9}$ Chez l'adulte, la plus grande calcification osseuse limite le guidage de l'aiguille en temps réel, mais dans l'espace lombaire, l'échographie peut être utile car un examen avant la procédure diminue le nombre d'essais de l'aiguille chez des patients ayant une anatomie difficile. ${ }^{10}$ Au niveau de la colonne thoracique moyenne de l'adulte, les espaces interlaminaires relativement étroits et les apophyses épineuses thoraciques fortement obliques limitent souvent la visibilité échographique des structures jusqu'à la duremère. Certains ont suggéré qu'une échographie de la colonne thoracique avant une procédure pouvait avoir un bénéfice clinique en précisant l'anatomie vertébrale, ${ }^{11}$ mais cet avantage a été réfuté dans une récente étude randomisée. $^{12}$ On a aussi montré récemment que le Doppler contribuait à la visualisation des cathéters périduraux placés dans l'espace péridural lombaire; néanmoins, l'efficacité de cette technique n'a pas été démontrée au niveau thoracique. ${ }^{13}$

L'analyse du tracé de la pression péridural a été utilisée pour confirmer la présence d'une aiguille ou d'un cathéter dans l'espace péridural. ${ }^{14}$ Sur le plan positif, cette technique est relativement simple et peu coûteuse à mettre en œuvre avec un équipement facilement disponible. Néanmoins, les facteurs régissant la relation entre le tracé péridural et la position du cathéter par rapport au nerf sont relativement obscurs et - dans certains cas le tracé peut être difficile à évaluer. L'analyse du tracé péridural ne permet pas non plus de différencier la position de l'extrémité du cathéter entre les espaces périduraux, intrathécaux et en intravasculaire. Enfin, la technique n'indique pas quel est le segment vertébral concerné, ou la latéralité de l'emplacement de l'extrémité du cathéter.

La dose test d'anesthésique local est considérée par beaucoup comme la norme de référence contre laquelle le bon positionnement d'un cathéter péridural doit être évalué. La dose test peut aussi contribuer, théoriquement, au diagnostic de cathéter inséré dans l'espace intrathécal ou en intravasculaire. Mais l'évaluation d'un bloc sensitif requiert la coopération du patient, après avoir donné au médicament le temps nécessaire pour agir et repose sur une réponse subjective du patient qui pourrait ne pas être exacte.

Comparé à ces autres modalités, l'EEST est relativement simple, efficace et portable et n'est associé à aucune exposition aux radiations pour les patients et les praticiens. L'EEST possède de grandes capacités diagnostiques et offre de nombreux avantages : il est sensible ${ }^{4-7}$; peut être rapidement administré; son résultat est reproductible et objectif (réponse évoquée motrice visible); il permet de savoir à quelle hauteur se situe l'extrémité du cathéter péridural; il peut différencier des cathéters positionnés en sous-cutané, intrathécal, ou en intravasculaire ${ }^{15}$; et pourrait contribuer à identifier un cathéter en position sous-dural ${ }^{16,17}$ (mais cela est controversé). ${ }^{18}$

À notre avis, les avantages de l'EEST en font un test supérieur pour la confirmation du bon positionnement d'un cathéter péridural. Quand les soins du patient sont transmis de l'anesthésiologiste de la salle d'opération à un autre médecin ou équipe de soins aigus postopératoires chargés de contrôler la douleur, il est particulièrement utile de disposer d'une évaluation objective documentée de la position du cathéter péridural au moment de sa pose. Mais alors si l'EEST est tellement utile, pourquoi 20 ans après sa description, n'est-il pas largement employé?

Nous supposons que de nombreux anesthésiologistes rechignent à reconnaître le taux élevé d'échecs d'analgésie péridurale décrit dans les publications et continuent de pratiquer avec la croyance erronée que la réussite d'une « technique de perte de résistance » et qu'un placement péridural sans incident du cathéter sont les signes suffisants de son bon positionnement. Un autre problème est la pression pour ne pas perdre de temps et faire avancer le cas, ce qui empêche l'adoption de tests permettant d'évaluer la position du cathéter péridural. Un problème propre à l'EEST est la disponibilité de l'équipement approprié. La majorité des stimulateurs nerveux, notamment, ne sont pas dotés des fonctionnalités nécessaires. Cela inclut une plage de courant de 0,1 à $15 \mathrm{~mA}$, des durées d'impulsion de 0,1 à $0,2 \mathrm{~ms}$, une fréquence d'impulsion de 1 à $2 \mathrm{~Hz}$, des afficheurs numériques de courant et des raccords appropriés (anode sur une électrode ECG, cathode sur un adaptateur ECG de Johan). Nous voudrions attirer l'attention des lecteurs sur le fait que la plage de courant requise pour évoquer une réponse motrice dans la description initiale $(1 \mathrm{à} 10 \mathrm{~mA})^{2}$ a été élargie à 1 à $15 \mathrm{~mA}^{19}$ En revanche, avec un peu d'effort, les cathéters périduraux utilisés habituellement peuvent servir pour l'EEST. Il est indispensable de créer une colonne continue de liquide conducteur dans le cathéter péridural en l'amorçant avec du salin après son insertion et de s'assurer qu'il n'y a pas d'air dans le système. ${ }^{3}$ Nous avons constaté que l'application d'une pression continue douce sur la colonne de salin au cours de la stimulation facilite une réponse motrice évoquée fiable et répétable. À noter que la majorité des études évaluant le test n'utilisent pas un cathéter de stimulation. ${ }^{3-5}$ En cas d'utilisation d'un tel cathéter péridural de stimulation, l'amorçage avec du salin n'est pas requis. ${ }^{20}$

L'EEST a été critiqué pour son incapacité à identifier le positionnement de l'extrémité du cathéter dans l'espace paravertébral. ${ }^{4}$ En dehors de la fluoroscopie continue avec péridurographie, aucune autre méthode n'est parvenue à résoudre ce problème à ce jour; biologiquement cependant, il paraîtrait plausible que l'analyse du tracé péridural présente de l'intérêt. Un autre problème à envisager est 
qu'un certain niveau d'analgésie péridurale peut quand même être atteint avec des cathéters situés en dehors de l'espace péridural si des volumes suffisants d'anesthésique local sont administrés, comme dans le cas des blocs du plan interfascia (par exemple, plan des muscles érecteurs du rachis, ${ }^{21}$ bloc rétrolaminaire ${ }^{22}$ et bloc du carré crural (quadratus lomburum)). ${ }^{23}$ Dans ce cas, l'incapacité à obtenir une réponse motrice évoquée au moyen de l'EEST ne correspondrait pas à un effet analgésique péridural et serait interprétée comme un «faux négatif ». Néanmoins, nous insistons sur le fait que l'EEST aurait correctement alerté sur le fait que le cathéter n'était pas inséré dans l'espace péridural.

Vingt ans après sa description initiale, l'EEST ou test de Tsui reste une technique objective, portable, fiable et sécuritaire pouvant contribuer à diminuer le taux élevé d'échecs des cathéters périduraux. Bien que l'adoption de l'EEST chez l'adulte paraisse plutôt lente, le besoin clinique de diminuer les échecs des blocs périduraux thoraciques est bien documenté. Nous espérons que l'étude de Balki et coll. ${ }^{3}$ rappellera aux cliniciens que l'EEST représente une option supérieure quand une confirmation du positionnement du cathéter péridural est recherchée. Nous sommes confiants et l'adoption en routine de l'EEST aidera à tirer le maximum des avantages de l'analgésie péridurale.

Conflict of interest Ban C. H. Tsui is the inventor of the Tsui test.

Editorial responsibility This submission was handled by Dr. Steven Backman, Associate Editor, Canadian Journal of Anesthesia.

Conflit d'intérêts Ban C. H. Tsui est l'inventeur du test de Tsui.

Responsabilité éditoriale Cet article a été traité par le $\mathrm{D}^{\mathrm{r}}$ Steven Backman, rédacteur adjoint, Journal canadien d'anesthésie.

\section{References}

1. Ready LB. Acute pain: lessons learned from 25,000 patients. Reg Anesth Pain Med 1999; 24: 499-505.

2. Tran DQ, Van Zundert TC, Aliste J, Engsusophon P, Finlayson $R J$. Primary failure of thoracic epidural analgesia in training centers: the invisible elephant? Reg Anesth Pain Med 2016; 41: 309-13.

3. Balki $M$, Malavade A, Ye XY, Tharmaratnam $U$. Epidural electrical stimulation test versus local anesthetic test dose for thoracic epidural catheter placement: a prospective observational study. Can J Anesth 2019; DOI: https://doi.org/10.1007/s12630019-01301-2.

4. Tsui BC, Gupta S, Finucane B. Confirmation of epidural catheter placement using nerve stimulation. Can J Anaesth 1998; 45: 640-4.

5. Forster JG, Niemi TT, Salmenpera MT, Ikonen S, Rosenberg PH. An evaluation of the epidural catheter position by epidural nerve stimulation in conjunction with continuous epidural analgesia in adult surgical patients. Anesth Analg 2009; 108: 351-8.
6. de Medicis E, Tetrault JP, Martin R, Robichaud R, Laroche L. A prospective comparative study of two indirect methods for confirming the localization of an epidural catheter for postoperative analgesia. Anesth Analg 2005; 101: 1830-3.

7. Tsui BC, Gupta S, Finucane B. Determination of epidural catheter placement using nerve stimulation in obstetric patients. Reg Anesth Pain Med 1999; 24: 17-23.

8. Tsui BC, Wagner A, Cave D, Kearney R. Thoracic and lumbar epidural analgesia via the caudal approach using electrical stimulation guidance in pediatric patients: a review of 289 patients. Anesthesiology 2004; 100: 683-9.

9. Lam DK, Corry GN, Tsui BC. Evidence for the use of ultrasound imaging in pediatric regional anesthesia: a systematic review. Reg Anesth Pain Med 2016; 41: 229-41.

10. Chin KJ, Perlas A, Chan V, Brown-Shreves D, Koshkin A, Vaishnav $V$. Ultrasound imaging facilitates spinal anesthesia in adults with difficult surface anatomic landmarks. Anesthesiology 2011; 115: 94-101.

11. Costache I, Ramlogan R, Chin KJ. The clinical benefits of ultrasound-guided thoracic epidural placement. Reg Anesth Pain Med 2017; 42: 793.

12. Auyong DB, Hostetter L, Yuan SC, Slee AE, Hanson NA. Evaluation of ultrasound-assisted thoracic epidural placement in patients undergoing upper abdominal and thoracic surgery: a randomized, double-blind study. Reg Anesth Pain Med 2017; 42: 204-9.

13. Elsharkawy H, Sonny A, Govindarajan SR, Chan V. Use of colour Doppler and M-mode ultrasonography to confirm the location of an epidural catheter - a retrospective case series. Can J Anesth 2017; 64: 489-96.

14. Elsharkawy H, Sonny A, Chin KJ. Localization of epidural space: a review of available technologies. J Anaesthesiol Clin Pharmacol 2017; 33: 16-27.

15. Tsui BC, Gupta S, Finucane B. Detection of subarachnoid and intravascular epidural catheter placement. Can J Anesth 1999; 46: 675-8.

16. Tsui BC, Gupta S, Emery D, Finucane B. Detection of subdural placement of epidural catheter using nerve stimulation. Can J Anesth 2000; 47: 471-3.

17. Lena $P$, Martin $R$. Subdural placement of an epidural catheter detected by nerve stimulation. Can J Anesth 2005; 52: 618-21.

18. Moore AR, Siddiqui N, Kassel EE, Carvalho JC. Unintentional subdural catheter placement during labor analgesia shows typical radiological pattern but atypical response to the Tsui test. Int $\mathbf{J}$ Obstet Anesth 2010; 19: 111-4.

19. Tsui BC. Epidural stimulation test criteria. Anesth Analg 2006; 103: 775-6.

20. Tsui BC, Usher A, Kulkarni PR, Scott SL. Thoracic epidural catheters via the caudal and lumbar approaches using styletted multiple port catheters in pediatric patients: a report of three cases. Acta Anaesthesiol Scand 2006; 50: 514-7.

21. Forero M, Rajarathinam M, Adhikary SD, Chin KJ. Erector spinae plane block for the management of chronic shoulder pain: a case report. Can J Anesth 2018; 65: 288-93.

22. Voscopoulos C, Palaniappan D, Zeballos J, Ko H, Janfaza D, Vlassakov $K$. The ultrasound-guided retrolaminar block. Can J Anesth 2013; 60: 888-95.

23. Sondekoppam RV, Ip V, Johnston DF, et al. Ultrasound-guided lateral-medial transmuscular quadratus lumborum block for analgesia following anterior iliac crest bone graft harvesting: a clinical and anatomical study. Can J Anesth 2018; 65: 178-87.

Publisher's Note Springer Nature remains neutral with regard to jurisdictional claims in published maps and institutional affiliations. 